\title{
Iris clipping of a diode laser beam when performing retinal photocoagulation
}

\author{
W H Woon, T J ffytche, A M P Hamilton, J Marshall
}

\begin{abstract}
The Microlase infrared diode laser has recently become available for retinal photocoagulation. It uses a laser beam with a large convergence angle $\left(23^{\circ}\right)$. This gives rise to a laser beam with a relatively large cross section at the subject's pupil. In this study the pupillary area required to view the fundus at the slit-lamp with a 90-dioptre lens is determined. It is found that the pupillary area required to view the fundus is smaller than the cross section of the Microlase laser beam at the pupil. Thus iris clipping of the laser beam can occur even though the surgeon has a clear and bright view of the fundus. Care should be taken to use the Microlase only in the presence of a widely dilated pupil.
\end{abstract}

The Microlase infrared diode laser (Keeler Ltd) has become available for clinical use in retinal photocoagulation. It has been shown to be effective for the treatment of proliferative disease and diabetic exudative retinopathy. ${ }^{1-3}$ Surgeons who are using the Microlase for the first time, and who have previous experience with argon lasers, need to adjust to the use of a system with a longer wavelength and a larger convergence angle. Argon laser photocoagulators emit at $488 \mathrm{~nm}$ and $514 \mathrm{~nm}$ and have a convergence angle of $7^{\circ}$ or less. In contrast, the Microlase diode laser emits at approximately $810 \mathrm{~nm}$ and has a convergence angle of $23^{\circ} .{ }^{4}$ The near infrared radiation used by the Microlase is not as well absorbed by melanin as the shorter wavelength used by argon laser photocoagulators. ${ }^{5}$ For this reason the Microlase requires higher operating powers than those used by argon lasers. ${ }^{1-3}$ The longer wavelength also results in deeper burns, which are grey in colour and less immediately visible than the white burns produced by argon lasers..$^{1-3}$

The surgeon using the Microlase should also be aware that the large convergence angle can lead to greater inadvertent variation in retinal irradiance than a laser system using a small convergence angle. The variation in retinal irradiance can arise from unintentional changes in retinal spot size and iris clipping of the laser beam. ${ }^{78}$ In most laser systems a larger spot size is achieved by progressively defocusing the treatment beam forward into the choroid. The Microlase achieves a larger spot size by defocusing the treatment beam back into the vitreous. This is performed by the spot size adjuster, which moves the Microlase relative to its base on the slit-lamp. Defocusing of the laser beam could be similarly achieved by keeping the Microlase fixed on its base and moving the slit-lamp away from the patient - that is, by increasing the working distance of the slit-lamp. This must be avoided if the spot size is to remain constant from one burn to the next.

The manufacturers recommend that the working distance of the slit-lamp be held constant by keeping the retina in sharp focus. ${ }^{4}$ However, inadvertent variation in the working distance of the slit-lamp can occur even if the retina is held in sharp focus owing to the depth of field of the operator's image and accommodation by the surgeon. Variation in spot size due to inadvertent variation in the working distance can also occur with argon lasers. ' However, the change in spot size for a given change in working distance is dependent on the convergence angle of the laser used. A small movement of the slit-lamp will cause a greater change in spot size if the convergence angle is large (Fig 1). Thus the spot size at the fundus is more sensitive to changes in the working distance when using the Microlase than when using an argon laser photocoagulator. When using the Microlase it is important to minimise the change in spot size which can occur from pulse to pulse by following the manufacturer's instructions to keep the retina in sharp focus.

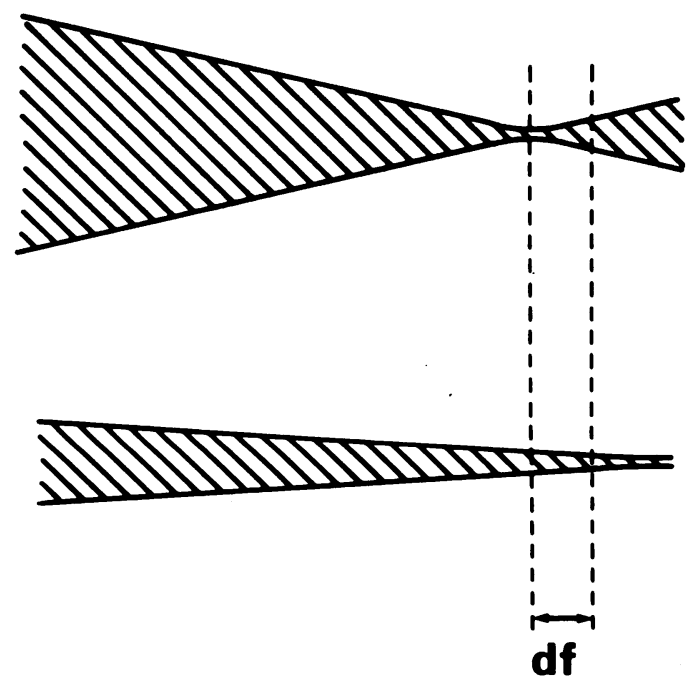

Figure 1 The laser spot size at the fundus depends on the position of the laser focus relative to the fundus. The top laser beam has a larger convergence angle than the bottom laser beam. There is a correspondingly larger change in spot size over the depth of field (df) of the retinal image.
Judd Street, London WCIH

Accepted for publication

19 February 1991 
Figure 2 A laser beam with a large convergence angle produces a wide laser beam at the subject's pupil.

Figure 3 Top. The slitlamp objective (SO) collects only light passing through a relatively small area of the subject's pupil. This area is called the viewing aperture. Bottom. The viewing apertures are the images of the slit-lamp objective $\left(\mathbf{I}_{\mathrm{s}}\right)$ formed by the

ophthalmoscopic lens (L).
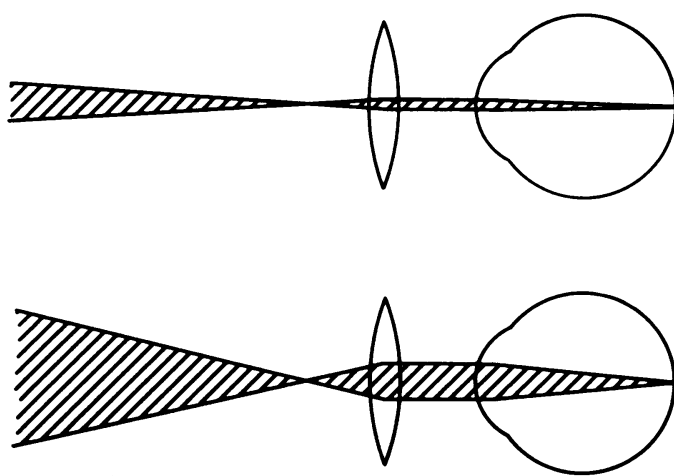

Iris clipping of the laser beam is not often encountered when using the argon laser photocoagulator owing to the relatively small size of the laser beam. ${ }^{9}$ The Microlase is more likely to result in iris clipping because a large convergence angle leads to a large laser beam diameter at the subject's pupil (Fig 2). ${ }^{8}$ When using an ophthalmoscopic lens to perform photocoagulation the pupil is not seen, and the operator cannot know whether the fundus is being viewed through the centre of the pupil. In certain circumstances iris clipping can occur without being apparent to the surgeon. For most laser photocoagulators the minimum spot size is achieved by focusing the laser on the fundus. In this situation iris clipping of the laser beam will not produce a similar clipping of the laser spot on the fundus, because the pupil of the eye is an aperture stop and not a field stop. ${ }^{10}{ }^{11}$ Iris clipping can also occur without changing the surgeon's view of the fundus if the laser beam cross section is larger than the pupillary area required to obtain a clear view of the fundus.

When the fundus is viewed through a slitlamp, the slit-lamp collects only light passing through a relatively small area of the subject's pupil. This area of the subject's pupil is called the viewing aperture. The viewing apertures are the images of the objectives of the slit-lamp formed by the ophthalmoscopic or contact lens (Fig 3). If the viewing apertures fit into an area smaller than the cross section of the laser beam at the pupil, then it would be possible for the laser beam to be clipped without affecting the view of the fundus.

We have measured the viewing aperture and compared this to the laser beam cross section at the cornea for the Microlase when using a 90 dioptre lens. The results are compared with measurements made for an argon laser photo-

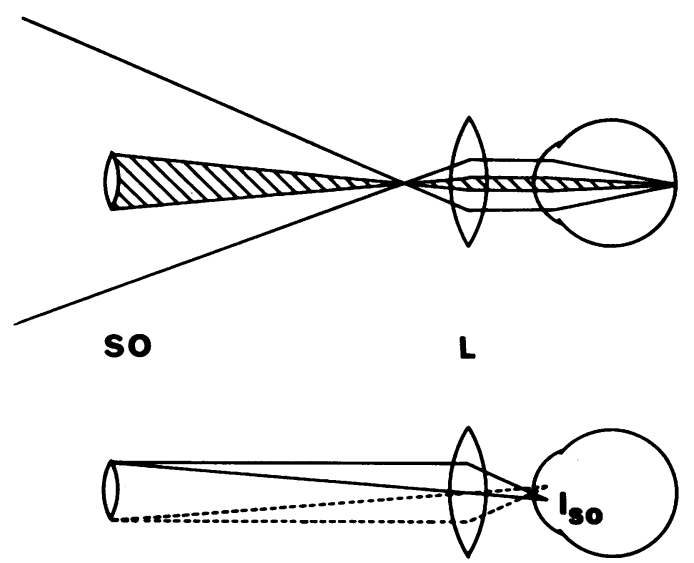

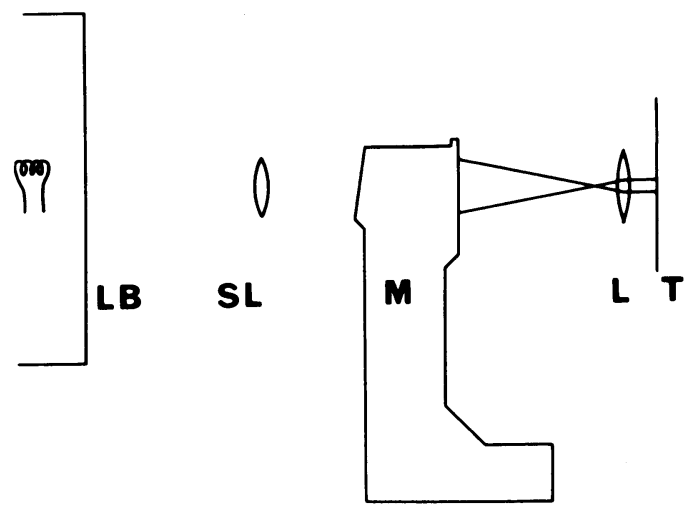

Figure 4 The viewing apertures may be determined by back illuminating the slit-lamp. LB: light box. SL: slit-lamp. M: Microlase. L: 90-dioptre lens. T: translucent screen.

coagulator. A model eye was then used to test whether iris clipping of the laser beam could occur without affecting the view of the fundus when the Microlase was used with a 90-dioptre lens. The results show that iris clipping may be a significant cause of variation in irradiance at the fundus and therefore the size and intensity of the resulting lesion.

\section{Materials and methods}

The dimensions of the viewing apertures when using a 90-dioptre lens were determined from the principle of reversibility of light rays. The slitlamp may be back illuminated through the eye pieces to determine the path of light rays from the subject's fundus through the ophthalmoscopic lens and slit-lamp (Fig 4). A Haag-Streit slitlamp with $10 \times$ eyepieces was used, and the viewing apertures were determined for both the $1 \times$ and the $1.6 \times$ objectives. A Haag-Streit slitlamp with a Microlase attached was adjusted so that the aiming beam of the Microlase was focused at the working distance of the slit-lamp to produce a minimum spot size of $100 \mu \mathrm{m} .{ }^{4}$ The working distance selector for the Microlase was set to give the short working distance required when using a 90-dioptre lens. ${ }^{4}$ A Volk 90-dioptre lens was positioned so that a collimated aiming beam was produced. This procedure would place the 90-dioptre lens in the position it would occupy when being used with an emmetropic

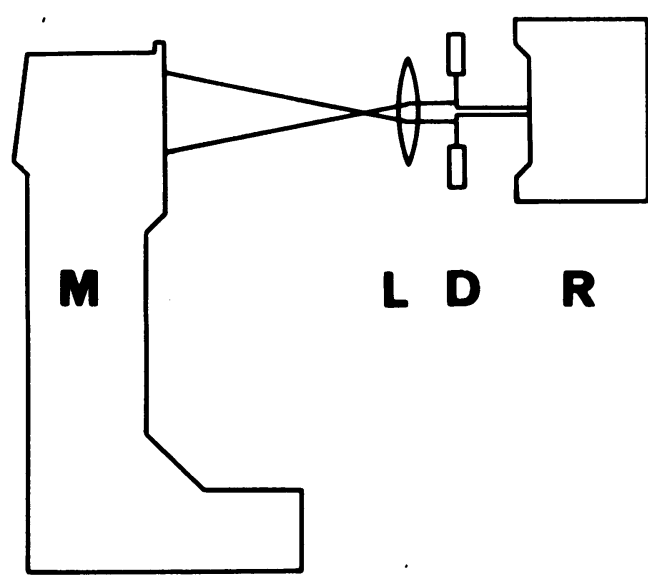

Figure 5 The beam diameter of the collimated Microlase laser beam may be determined using a variable diaphragm. M: Microlase. L: 90-dioptre lens. D: variable diaphragm. $\mathrm{R}$ : radiometer. 


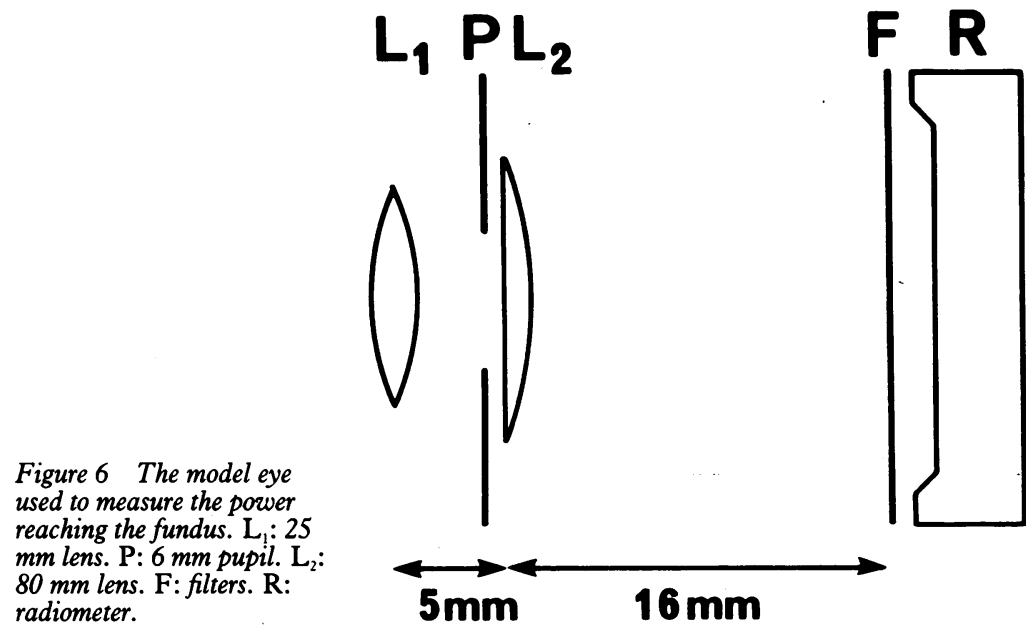

eye. The slit-lamp was back illuminated with a diffuse extended source (a lightbox). A thin translucent screen with a $1 \mathrm{~cm}$ calibration scale was positioned to be coincident with the image of the viewing apertures. The screen was photographed to record the dimensions of the viewing apertures. The approximate dimensions of the aiming beam could be recorded at the same time. The procedure was repeated for a coherent system 900 argon laser photocoagulator and Zeiss slit-lamp. The Zeiss slit-lamp was set at magnification 12 and the laser beam spot size was set at $100 \mu \mathrm{m}$.

The beam diameter of the Microlase infrared treatment beam, after being collimated by a 90 dioptre lens, was separately determined (Fig 5). A variable diaphragm was placed in the approximate plane of the pupil, and the diaphragm was centred on the diode beam by means of the inbuilt aiming beam of the Microlase. The power, from the laser, passing through the diaphragm was determined for a range of aperture settings with a radiometer (United Detector Technology Model S370), adjusted for the wavelength of the Microlase.
Table 1 Dimensions of viewing apertures

\begin{tabular}{lcc}
\hline $\begin{array}{l}\text { Slit-lamp and } \\
\text { objective }\end{array}$ & $\begin{array}{l}\text { Viewing aperture } \\
\text { diameter, } \mathrm{mm}\end{array}$ & $\begin{array}{l}\text { Separation of centre } \\
\text { of viewing apertures, } \\
\mathrm{mm}\end{array}$ \\
\hline Haag-Streit $\times 1$ & 1.2 & 2.5 \\
Haag-Streit $\times 1.6$ & 1.8 & 2.5 \\
Argon $\times 12$ & 1.2 & 2.5 \\
\hline
\end{tabular}

To test whether a significant amount of laser energy could be blocked without altering the view of the fundus, a model eye was used incorporating a radiometer in the plane of the fundus (Fig 6). The aperture used to mimic the anatomical pupil was $6 \mathrm{~mm}$ in diameter. The radiometer (United Detector Technology Model S370) was used to measure the power of each pulse from the Microlase. A Kodak Wratten 87C filter was employed to block out light with wavelength shorter than $790 \mathrm{~nm}$, and a neutral density filter was used to protect the radiometer. The Haag-Streit slit-lamp had the $1.6 \times$ objectives in place and the 90-dioptre lens was positioned in front of the model eye to obtain a view of the centre of the filters. The laser power reaching the radiometer was measured for a fixed laser power setting and for a fixed axial location of the 90-dioptre lens, but with varying degrees of lateral displacement of the lens while retaining the central $6 \mathrm{~mm}$ of the radiometer in the field of view through the slit-lamp.

\section{Results}

The viewing apertures and the laser beam cross section recorded when back illuminating the slitlamp are shown in Figure 7. The dimensions are tabulated in Table 1. All of the energy of the Microlase treatment beam, after it has been collimated by a 90 -dioptre lens, passes through a cross section of $5 \mathrm{~mm}$ diameter (Fig 8, Table 2). $87 \%$ of the energy passes through a cross section of $4 \mathrm{~mm}$ diameter.

When using the Microlase with the model eye
Figure 7 Photographs of the viewing apertures and laser beam cross section recorded when back illuminating the slit-lamp. The scale has intervals of $\mathrm{mm}$. Top left: Viewing aperture of a Zeiss slit-lamp at magnification 12. The argon laser beam lies between the two viewing apertures. Top right: Cross section of Microlase aiming beam and viewing apertures of a Haag-Streit slit-lamp with $1 \times$ objectives. The viewing apertures lie within the cross section of the Microlase aiming beam. Bottom left: Viewing apertures of a Haag-Streit slit-lamp with $1 \times$ objectives. The viewing apertures lie either side of the image of the slit-lamp mirror. Bottom right: Viewing apertures of $a$ Haag-Streit slit-lamp with $1.6 \times$ objectives. The viewing apertures lie either side of the image of the slitlamp mirror.

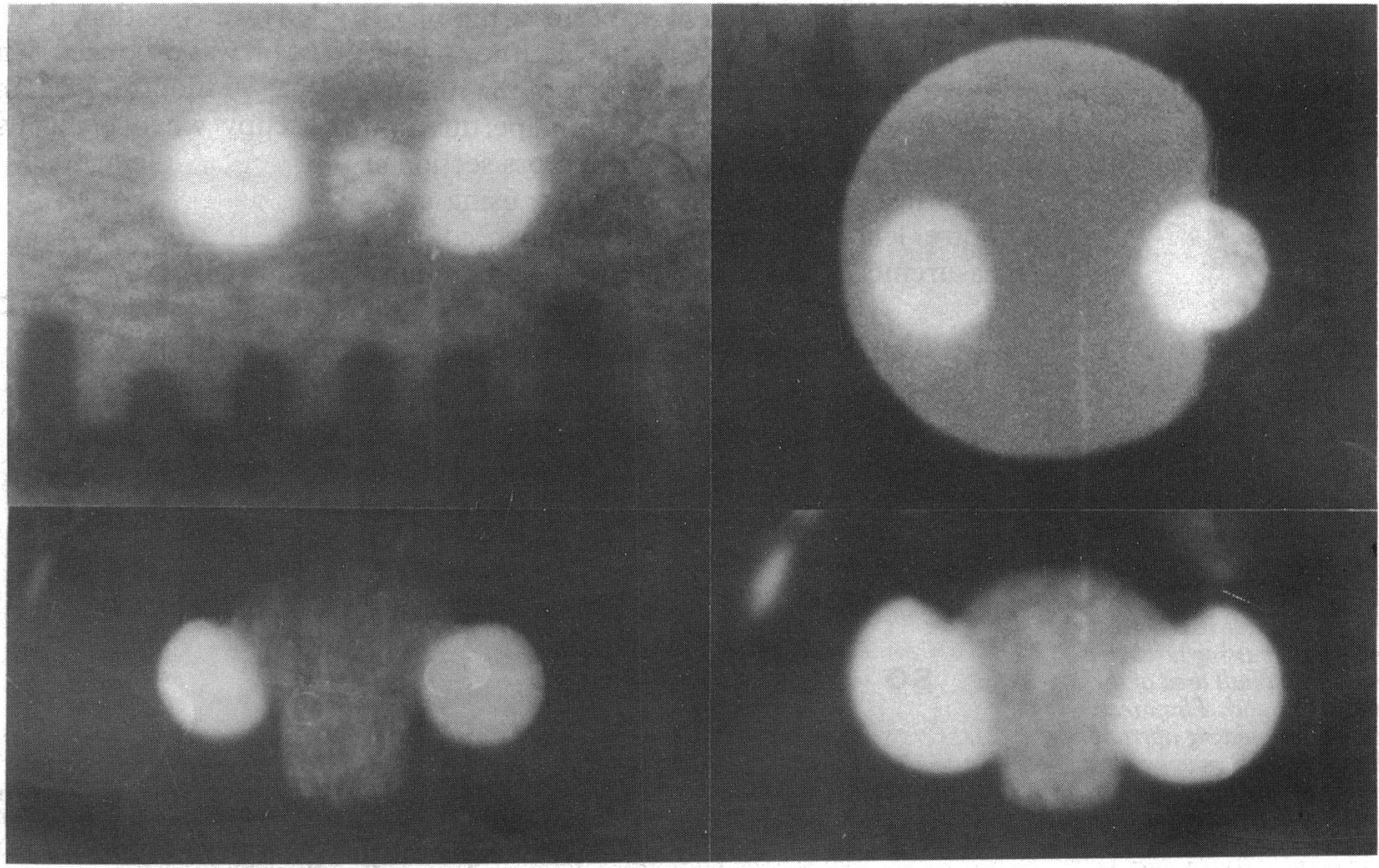


Figure 8 Plot of laser power passing through the diaphragm versus diaphragm aperture diameter, for the Microlase treatment beam collimated by a 90-dioptre lens.

Table 2 The percentage power contained in the central $\mathrm{d} \mathrm{mm}$ of the Microlase treatment beam collimated by a 90-dioptre lens

\begin{tabular}{lc}
\hline Diameter, $d$ mm & Power \% \\
\hline 5 & 100 \\
4 & 87 \\
3 & 55 \\
2 & 27 \\
1 & 8 \\
\hline
\end{tabular}

Figure 9 Viewing apertures and laser beam cross sections at the cornea. The Microlase laser beam may be clipped (hatched area) without clipping the viewing apertures. M: $4 \mathrm{~mm}$ laser beam. $\mathrm{V}_{1}$ : viewing aperture of Haag-Streit slitlamp with $1 \times$ objective. $\mathrm{V}_{2}$ : viewing aperture of HaagStreit slit-lamp with $1 \cdot 6 \times$ objective. A: argon laser beam. $V_{3}$ : viewing aperture of Zeiss slit-lamp with $12 \times$ magnification.
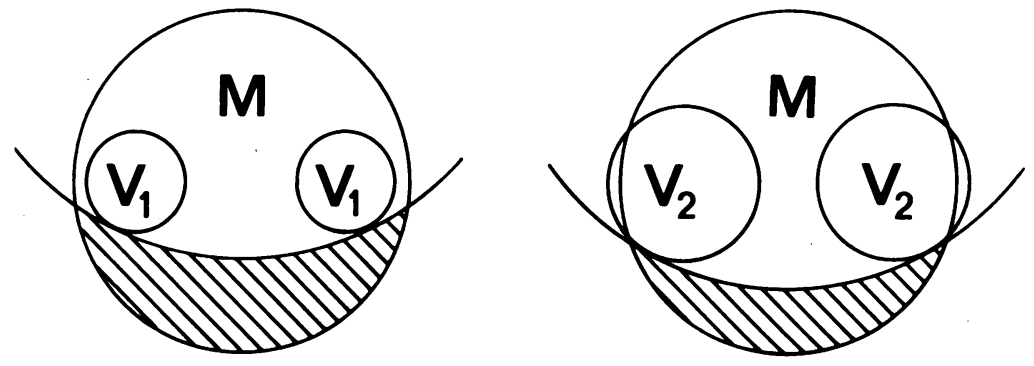

it was found that the power reaching the fundus in the model eye could vary by $20 \%$ by moving the 90 -dioptre lens $\pm 5 \mathrm{~mm}$ vertically without subjectively altering the view of the fundus. For any given position of the 90-dioptre lens there was no variation of the power reaching the radiometer.

\section{Discussion}

It has been shown, by means of a model eye, that when the Microlase is used with a 90 -dioptre lens a $20 \%$ variation in the power at the fundus can occur from pulse to pulse. This variation is probably due to iris clipping of the laser beam. When used with a 90-dioptre lens, the Microlase gives rise to a laser beam with a diameter of between 4 and $5 \mathrm{~mm}$. This is larger than the vertical dimensions of the viewing apertures (Fig 9), and thus the laser beam may be clipped by the iris without clipping of the viewing apertures. In comparison, the argon laser beam is smaller than an individual viewing aperture and lies between the two viewing apertures (Fig 9). In this situation it would not be possible to clip the laser beam without clipping the viewing apertures. The argon laser beam may be clipped by partially occluding the viewing apertures while retaining the same field of view, but this is less likely to occur than clipping of the Microlase laser beam.

The viewing apertures and laser beam diameters have been measured without taking into account the effect of refraction by the cornea. However, these results may be compared to the pupil size by using the entrance pupil rather than the anatomical pupil. The imaginary entrance pupil is the image of the anatomical pupil formed by the cornea, and it is 1.15 times the size of the anatomical pupil. ${ }^{12}$ The pupil size that is measured without taking into account refraction by the cornea is the size of the entrance pupil. When using a 90-dioptre lens, the surgeon cannot know that the laser beam is passing through the centre of the pupil, and iris clipping of the Microlase laser beam may occur even with the most widely dilated pupil. For a $7 \mathrm{~mm}$ entrance pupil $17 \%$ of the area of a $4 \mathrm{~mm}$ laser beam may be clipped without clipping the viewing apertures when using the $1 \cdot 6 \times$ objectives, and this may rise to $27 \%$ for the $1 \times$ objectives. The fraction of energy lost due to iris clipping will be less than the fraction of the area of the beam occluded because of the fall in beam energy distribution away from the central axis of the laser beam. However, up to $50 \%$ of the laser beam area can be occluded without significantly affecting the surgeon's view other than its becoming progressively dimmer. This is because the viewing apertures themselves are entrance pupils, and partially occluding them would reduce the brightness of the fundal image but not the field of view.

The authors have noted that, when using the Microlase, the lesion size for a given power setting shows more variability than when using argon lasers. It is possible that this is due to clipping of the laser beam by the iris. However, other causes of unintentional variation in retinal irradiance, such as accommodation by the surgeon and the depth of field of the retinal image, may also be important. ${ }^{7}$ Uncontrolled variation in the retinal irradiance may be clinically more significant when near infrared lasers are used. A narrow power range for producing a satisfactory lesion in any individual patient has been observed in clinical practice. ${ }^{23}$ This narrow power range may be more apparent than real. An apparently narrow power range for optimum treatment will be observed if it is difficult to judge the intensity of the burn by its appearance.

When performing retinal photocoagulation with the argon laser, the laser burns are white and are seen immediately in high contrast against the background. Titrating the power level to produce a satisfactory burn is then relatively easy. However, with near infrared radiation the optimum burn is grey, takes time to become visible, and is seen in poor contrast against the background. ${ }^{1}$

These factors, of both visibility and latency, make it relatively difficult to titrate the power of a near infrared laser against the intensity of the burn. In this situation unintentional variation in retinal irradiance would be especially dangerous if the power had been set near the upper level of the range for optimum treatment in the presence of iris clipping.

The principles demonstrated with a 90-dioptre lens also apply to other ophthalmoscopic or contact lenses. The surgeon should obtain as
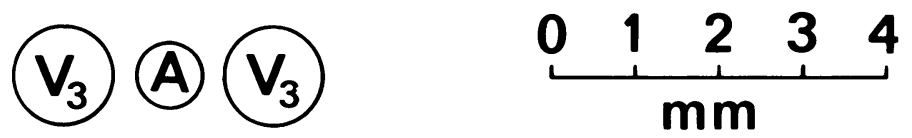
widely dilated a pupil as possible when using the Microlase. However, iris clipping is still possible with the most widely dilated pupil, and may occur because of the difficulty in ensuring that photocoagulation is taking place through the centre of the pupil. In cases where the pupil cannot be dilated widely, the surgeon should consider using a laser with a smaller convergence angle.

Postscript. The laser beam characteristics of the Microlase have been modified since this study was performed. However, the convergence angle in the vertical plane remains at $23^{\circ}$ and the problems demonstrated in this study remain.

The authors thank Keeler Ltd for supplying a Microlase infrared aser photocoagulator and for helpful discussion concerning its optical system. We are grateful to the Allerton Fund for providing the Allerton research fellowship to support $W$ H Woon.

1 McHugh JDA, Marshall J, ffytche TJ, Hamilton AM, Raven $A$, Keeler CR. Initial clinical experience using a diode laser in the treatment of retinal vascular disease. Eye 1989; 3: 51627.

2 Noyori K, Noyori S, Ohki R. Clinical trial of a diode laser photocoagulator-a preliminary report. Laser Light Ophthalmol 1990; 3: 81-7.

3 Brancato R, Bandello F, Trabucchi G, Leoni G, Lattanzio R. Argon and diode laser photocoagulation in proliferative diabetic retinopathy: a preliminary report. Laser Light Ophthalmol 1990; 3: 233-7.

4 Microlase user guide. Windsor: Keeler Ltd, 1989.

5 Mainster MA. Wavelength selection in macular photocoagulation. Ophthalmology 1986; 93: 952-8.

tion. Ophthalmology 1986; 93: 952-8.
6 McHugh JDA, Marshall J, Capon M, Rothery S, Raven A, Naylor RP. Transpupillary retinal photocoagulation in the eyes of rabbit and human using a diode laser. Laser Light Ophthalmol 1988; 2: 125-43.

7 Pomerantzeff $\mathrm{O}$, Schepens CL. Variation of energy density in argon laser photocoagulation. Arch Ophthalmol 1990; 93: 1033-5.

8 Mainster MA, Sliney DH, Belcher CD, Buzney SM. Laser photodisruptors. Ophthalmology 1983; 90: 973-91.

9 L'Esperance FA. Complications. In: L'Esperance FA, ed. Ophthalmic lasers. St Louis: Mosby, 1989; 2: 976.

10 Stenstrom S. Optics and the eye. London: Butterworth, 1964: 106.

11 Pedrotti FL, Pedrotti LS. Introduction to optics. New Jersey: Prentice-Hall, 1987; chapter 6: 106-10.

12 Bennett AG, Francis JL. The eye as an optical system. In: Davson H, ed. The Eye. New York: Academic Press, 1962; 4: 106. 